\title{
Design and Rationale of a Randomized, Double-Blind, Placebo-Controlled, Phase 2/3 Study Evaluating Dociparstat in Acute Lung Injury Associated with Severe COVID-19
}

\author{
Joseph A. Lasky · Jyotsna Fuloria · Marion E. Morrison (D) · \\ Randall Lanier $\cdot$ Odin Naderer $\cdot$ Tom Brundage $\cdot$ Allen Melemed
}

Received: September 14, 2020 / Accepted: October 14, 2020 / Published online: October 27, 2020

(C) The Author(s) 2020

\begin{abstract}
Introduction: The COVID-19 global pandemic caused by the novel coronavirus, SARS-CoV-2, and the consequent morbidity and mortality attributable to progressive hypoxemia and subsequent respiratory failure threaten to overrun hospital critical care units globally. New agents that address the hyperinflammatory "cytokine storm" and hypercoagulable pathology seen in these patients may be a promising approach to treat patients, minimize hospital stays, and ensure hospital wards and critical care units are able to operate effectively. Dociparstat sodium (DSTAT) is a glycosaminoglycan derivative of heparin with robust anti-inflammatory properties, with the potential to address underlying
\end{abstract}

Electronic supplementary material The online version of this article (https://doi.org/10.1007/s12325020-01539-z) contains supplementary material, which is available to authorized users.

J. A. Lasky

Tulane University Medical Center, New Orleans, LA, USA

J. Fuloria

University Medical Center New Orleans, New

Orleans, LA, USA

M. E. Morrison $(\bowtie) \cdot$ R. Lanier · O. Naderer .

T. Brundage $\cdot$ A. Melemed

Chimerix, Inc, Durham, NC, USA

e-mail: mmorrison@chimerix.com;

clinicaltrials@chimerix.com causes of coagulation disorders with substantially reduced risk of bleeding compared to commercially available heparin.

Methods: This study is a randomized, doubleblind, placebo-controlled, phase $2 / 3$ trial to determine the safety and efficacy of DSTAT added to standard of care in hospitalized adults with COVID-19 who require supplemental oxygen. Phase 2 will enroll 12 participants in each of two dose-escalating cohorts to confirm the safety of DSTAT in this population. Following review of the data, an additional 50 participants will be enrolled. Contingent upon positive results, phase 3 will enroll approximately 450 participants randomized to DSTAT or placebo. The primary endpoint is the proportion of participants who survive and do not require mechanical ventilation through day 28 . Discussion: Advances in standard of care, recent emergency use authorizations, and positive data with dexamethasone have likely contributed to an increasing proportion of patients who are surviving without the need for mechanical ventilation. Therefore, examining the time to improvement in the NIAID score will be essential to provide a measure of drug effect on recovery. Analysis of additional endpoints, including supportive biomarkers (e.g., IL-6, HMGB1, soluble-RAGE, D-dimer), will be performed to further define the effect of DSTAT in patients with COVID-19 infection.

Trial Registration: ClinicalTrials.gov identifier; NCT04389840, Registered 13 May 2020 
Keywords: Acute lung injury; COVID-19; Dociparstat; HMGB1; NETs; Neutrophil extracellular traps; P-selectin; Platelet factor 4

\section{Key Summary Points}

Dociparstat (DSTAT) is a heparin derivative with significantly decreased anticoagulant activity; this enables dosing at higher concentrations to achieve antiinflammatory effects that cannot be safely achieved with fully anticoagulant heparins

DSTAT's mechanism of action is multimodal-by inhibiting several key proteins (HMGB1, Platelet Factor 4, P-selectin) involved in the hyperinflammation and coagulopathy that contribute to high morbidity and mortality in severe COVID-19 DSTAT is uniquely positioned as a candidate treatment to address these important aspects of COVID-19 pathophysiology

This study aims to generate meaningful data to potentially advance the treatment of severe COVID-19 through scientific rigor and the use of a double-blind, randomized, placebo-controlled study design

\section{DIGITAL FEATURES}

This article is published with digital features, including a summary slide, to facilitate understanding of the article. To view digital features for this article go to https://doi.org/10.6084/ m9.figshare.13079213.

\section{INTRODUCTION}

Novel coronavirus 2019 disease (COVID-19) is caused by infection with severe acute respiratory syndrome coronavirus 2 (SARS-CoV-2). Clinical manifestations of COVID-19 range from mild, self-limited respiratory tract illness to severe alveolar damage leading to progressive respiratory failure, multiple organ failure, and death [1-3]. Approximately 15-20\% of COVID19 patients develop severe symptoms and exhibit systemic hyperinflammation with elevated cytokine levels and lung immune cell infiltration, which may result in acute damage to capillaries and lung epithelia/alveoli [4]. Additional localized pulmonary thrombotic microangiopathy and a low-grade disseminated intravascular coagulopathy (DIC) add to the lung pathology and organ dysfunction, increasing the risk of death $[5,6]$.

While the use of anticoagulants such as heparins may have potential clinical benefit [7], they come with the risk of major bleeding events [8]. The global health crisis caused by SARS-CoV-2 mandates aggressive development of both preventative vaccines and therapeutic regimens that effectively treat patients. Current efforts to expedite availability of therapeutics with a demonstrated positive risk/benefit profile include repurposing approved drugs or those already in advanced development for other indications. Dociparstat sodium (DSTAT; 2-O, 3-O desulfated heparin) is one such agent that is currently in late-stage development for acute myeloid leukemia. DSTAT is made by selective removal of the 2 -sulfate group from iduronic acid 2-sulfate and the 3 '-sulfate group from 3,6 bis-sulfated glucosamine present in unfractionated heparin. Removal of these sulfates is associated with significantly reduced anticoagulant activity while maintaining the known anti-inflammatory activities of heparin [9].

DSTAT inhibits the activity of high mobility group box protein 1 (HMGB1), a cytokine that plays a major role in the pathogenesis of immune disorders. Elevated HMGB1 in serum is strongly associated with clinical severity and mortality in COVID-19 patients [10]. Extracellular HMGB1, released from dying cells or secreted by activated immune cells, interacts with the receptor for advanced glycation end products (RAGE) and Toll-like receptors (TLR), causing the release of proinflammatory cytokines including, but not limited to, interleukin- 6 (IL-6), tumor necrosis factor-alpha (TNF- $\alpha$ ), monocyte chemoattractant protein-1 (MCP-1), 
and macrophage inflammatory protein-1 alpha $(\mathrm{MIP}-1 \alpha)$, all of which are elevated in severe COVID-19 [9, 11-13].

Infiltration of monocytes and other immune cells into the inflamed lung tissue is also an important pathogenic driver of acute lung injury. In vivo disease models have demonstrated that DSTAT can reduce HMGB1 in bronchoalveolar lavage fluid, decrease immune cell infiltration in lungs, and improve survival [14-16]. Molecular drivers of these effects likely include DSTAT inhibition of HMGB1 binding to RAGE, inhibition of leukocyte lung infiltration via decreases in MCP-1 expression, and direct blocking of the cell adhesion molecule P-selectin $[9,17,18]$.

COVID-19 coagulopathies may be attributed to several mechanisms including aberrant activation of immune cell responses, excessive inflammation, and immunothrombosis. For example, neutrophils are early responders to infection capable of extruding granular and nuclear contents to produce neutrophil extracellular traps (NETs). While NETs are often beneficial in trapping and eliminating pathogens, they can also promote thrombosis and are correlated with intubation and death in COVID-19 [19-21]. HMGB1 and platelet factor 4 (PF4), which are both significantly elevated in COVID-19 patients, induce formation of NETs and regulate NET degradation. DSTAT inhibition of HMGB1 and PF4 activities may reduce formation of NETs and promote their clearance $[9,22]$.

A related pathologic feature of COVID-19 is platelet hyperreactivity, which may contribute to NET formation and life-threatening coagulopathies. In severe disease, platelets have increased expression of P-selectin, which promotes the formation of platelet-leukocyte aggregates. These aggregates are associated with platelet hyperreactivity, immunothrombosis, and damage to lung capillaries [23]. Notably, P-selectin neutralization inhibits development of acute lung injury in animal models; DSTAT effectively blocks the binding and cell adhesion activities of P-selectin [9].

Overall, DSTAT inhibits several potentially key molecular drivers of COVID-19 pathology, including HMGB1, PF4, and P-selectin (Fig. 1).
DSTAT's pharmacologic mechanisms hold the potential to reduce both inflammation and coagulopathies, which contribute to the high morbidity and mortality in hospitalized COVID-19-infected patients population requiring supplemental oxygen. This study aims to determine if DSTAT provides added benefit to standard of care in COVID-19 patients at risk of acute lung injury by decreasing their need for ventilatory support and improving overall clinical outcomes.

\section{METHODS}

\section{Study Design}

Chimerix protocol CMX-DS-004 is a randomized, double-blind, placebo-controlled, phase $2 / 3$ study to determine the safety and efficacy of DSTAT versus placebo in adults receiving standard of care for acute lung injury associated with severe COVID-19 who are at high risk of respiratory failure.

This trial is designed in accord with the consensus ethical principles derived from international guidelines including the Declaration of Helsinki and Council for International Organizations of Medical Sciences (CIOMS) International Ethical Guidelines; applicable International Council of Harmonization (ICH) Good Clinical Practices (GCP) Guidelines; and applicable laws and regulations. All documents are initially approved by Advarra central institutional review board (IRB) and/or by the individual site IRBs as required (see Table S1 in Supplementary materials). Written informed consent is obtained from all participants before any study-related procedures are implemented. When available, the results will be published in a peer-reviewed journal.

Participants are randomized 1:1 in three sequential phase 2 cohorts, followed by the phase 3 portion of this pivotal study (Fig. 2). Randomization of participants in phase 2/cohort 3 and phase 3 will be stratified by baseline score on the National Institute of Allergy and Infectious Diseases (NIAID) ordinal scale (3 or 4 ) and by age ( $<60$ or $\geq 60$ years) [24]. Randomization of participants in phase 3 will 


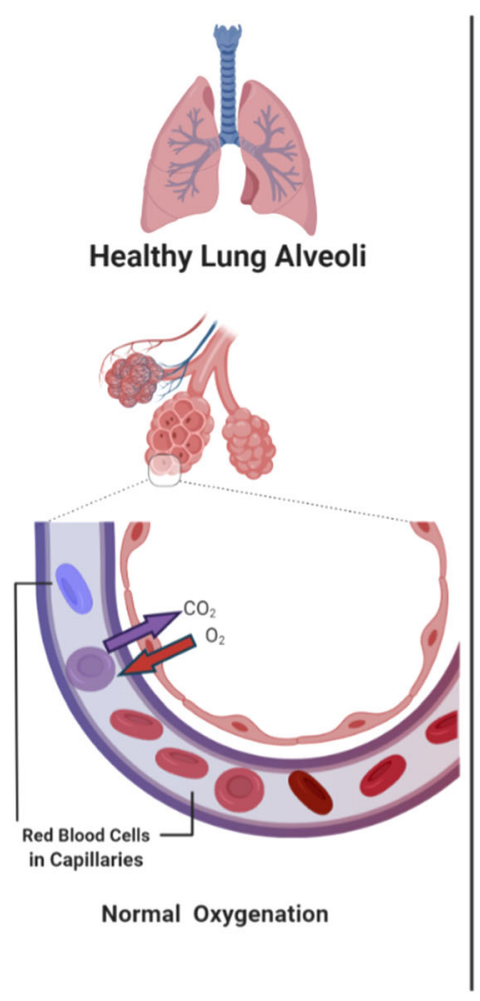

Fig. 1 Potential of DSTAT in COVID-19. Moderate-tosevere COVID-19 manifests with excessive inflammation, infiltration of activated immune cells into the lungs and coagulation disorders (e.g., clot formation) in the blood and tissues. DSTAT has the potential to prevent and mitigate these severe effects of SARS-CoV-2 infection by

additionally be stratified by baseline body mass index (BMI), i.e., $<34$ or $\geq 34 \mathrm{~kg} / \mathrm{m}^{2}$.

The primary objective of phase 3 is to assess the effect of dociparstat on disease progression in participants with severe COVID-19. Approximately 15 clinical sites in the USA are to be engaged in phase 2 to enroll a total of 74 participants. Phase 3 is expected to include approximately 75 clinical sites to enroll 452 participants.

\section{Eligibility}

Eligible participants must be adults between the ages of 18 and 85 years, who are hospitalized for laboratory-documented COVID-19 (e.g., positive SARS-CoV-2 nasopharyngeal swab by reverse transcriptase polymerase chain reaction
[RT-PCR]), with a resting oxygen saturation on room air of $\leq 93 \%$, and have a score of 3 or 4 on the NIAID ordinal scale (i.e., hospitalized requiring supplemental oxygen).

Key exclusion criteria include the requirement for invasive mechanical ventilation or extracorporeal membrane oxygenation (ECMO), acute pericarditis, severe renal impairment (i.e., calculated creatinine clearance $<30 \mathrm{ml} / \mathrm{min}$ ), an irreversible disease or condition with a mortality of $\geq 50 \%$ within 6 months, the presence or risk of active or uncontrolled bleeding, or a pre-existing history of severe chronic respiratory disease, severe chronic liver disease, or congestive heart failure requiring hospitalization. Eligible participants must not be receiving chronic anticoagulation, treatment with non-steroid immunomodulators 


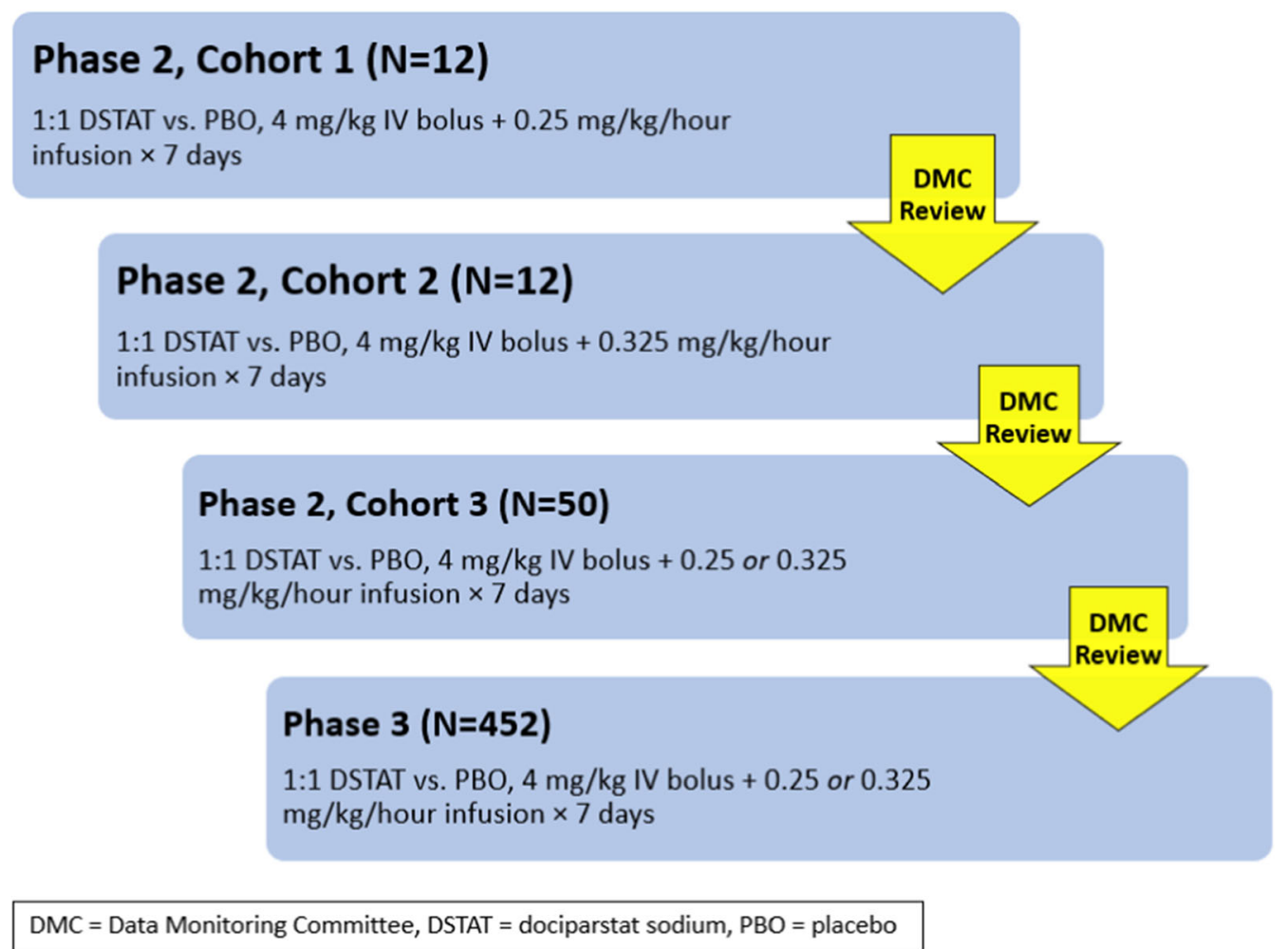

Fig. 2 CMX-DS-004 study schematic. All participants receive randomized treatment for up to 7 days and are followed through day 28 . The continuous infusion dose administered in phase 2 , cohorts 2 and 3 will be confirmed

or immunosuppressant medications, or other investigational or non-approved therapies for COVID-19. Laboratory exclusion criteria include an activated partial thromboplastin time $($ aPTT $)>42 \mathrm{~s}$, alanine aminotransferase or aspartate aminotransferase $>5$ times the upper limit of normal, or a platelet count $<80,000 /$ $\mathrm{mm}^{3}$.

Importantly, due to the risk of thromboembolic events in COVID-19, participants are permitted to receive prophylaxis for deep venous thrombosis with subcutaneous administration of enoxaparin or unfractionated heparin up to intermediate intensity, with dosing based on BMI and creatinine clearance. after review of data by an independent Data Monitoring Committee (DMC) from prior cohort(s). The phase 3 continuous infusion dose will be the same as administered in phase 2 , cohort 3

\section{Interventions}

Participants will receive blinded study intervention consisting of either DSTAT or normal saline as a placebo control for up to 7 days. Both groups will also receive best supportive care, as determined by the investigator. All participants will be followed through day 28 .

\section{Safety Considerations}

Based on DSTAT's relationship to heparin with its pharmacologically mediated effect on coagulation and results of nonclinical and clinical studies possible risks of particular interest with DSTAT therapy include prolonged aPTT (with a potential associated risk of bleeding/hemorrhagic events) and an increase in serum aminotransferase concentrations. In previous 
studies in which higher doses of DSTAT have been administered, mild to modest aPTT elevations have been observed, which were manageable with monitoring, and DSTAT dose adjustment, if needed. The transient elevation of liver enzymes is considered a non-adverse class effect for all heparins, heparin derivatives, low-molecular-weight heparins, and heparinoids [25]. Nevertheless, in the interest of subject safety, eligibility criteria have been designed to ensure subjects enrolled in the study are not at increased bleeding risk based on pre-existing conditions, and enrolled subjects will have coagulation tests performed at baseline and throughout study drug infusion. A conservative aPTT threshold for study drug interruption (i.e., $>50 \mathrm{~s}$ ) has been included in the protocol, with resumption of dosing allowed only if aPTT decreases to $<40 \mathrm{~s}$. Subjects who receive enoxaparin will also have anti$\mathrm{Xa}$ levels monitored to maintain levels of $<0.6 \mathrm{IU} / \mathrm{ml}$.

Notably, both unfractionated and lowmolecular-weight heparins have been associated with heparin-induced thrombocytopenia (HIT). In contrast, DSTAT suppresses heparininduced thrombocytopenia-related platelet activation in vitro and may disrupt formation of heparin/PF4 multimolecular complexes [26].

\section{Measurements}

Efficacy will be evaluated through clinical examinations, oxygen requirements, and laboratory tests.

Safety will be monitored through collection of adverse event data and laboratory samples.

Plasma samples will be collected for analysis of DSTAT concentrations and biomarkers to further elucidate the mechanism of action of DSTAT, and sputum samples will be collected for virology testing.

\section{Endpoints}

The primary efficacy endpoint is the proportion of participants who are alive and do not require invasive mechanical ventilation through day 28. Secondary efficacy endpoints include all- cause mortality, time to clinical improvement defined as at least a 2-grade improvement on the NIAID ordinal scale, number of ventilatorfree days, time to hospital discharge, and average daily corticosteroid use. Dociparstat plasma concentrations and pharmacokinetic parameters will be summarized, as will biomarkers related to the pathophysiology of severe COVID-19, including HMGB1, soluble RAGE, IL-6, and TNF $\alpha$.

Safety endpoints include incidence of adverse events, changes from baseline in clinical laboratory parameters, and distribution of graded clinical laboratory results.

\section{Sample Size and Power Calculations}

Approximately 600 potential participants are expected to be screened to achieve a total of approximately 74 participants randomized in phase 2 and 452 participants randomized in phase 3.

The sample size of six participants per treatment group for cohorts 1 and 2 was selected to assess any safety signals in a limited number of participants. The sample size for cohort 3 was selected to provide a reasonable level of precision to assess the treatment effect for the primary endpoint.

For phase 3, the sample size of 226 participants per group was selected to detect the difference between a control failure rate of $20 \%$ and a dociparstat failure rate of $10 \%$ at a twosided 0.05 alpha level with $>80 \%$ power. This sample size also accounts for a futility analysis after $50 \%$ of the phase 3 participants are enrolled using a Pocock beta spending function. Participants enrolled in phase 2 will not contribute to the inferential phase 3 analysis.

\section{Statistical Methodology}

The primary phase 3 analysis will utilize the intent-to-treat analysis set and a CochranMantel-Haenszel test stratified by each of the actual stratification factors at the 0.05 alpha level. Participants will be counted as failures once they go on a ventilator or die. Number and percentage of failures will be presented for each 
treatment arm. Cochran-Mantel-Haenszel $p$ values, estimated common odds ratios, estimated common risk differences, and corresponding approximate two-sided 95\% confidence intervals will be presented. The Breslow-Day test will be used to test the homogeneity of the odds ratios across strata. Although missing data are expected to be limited, any such cases will be imputed as failure. A supportive analysis using multiple imputation will be done to assess the sensitivity of the inference to the approach to missing data.

In the event the primary endpoint is met, the key secondary analysis of all-cause mortality at day 28 will be tested sequentially at the 0.05 alpha level, using the same method used for the primary endpoint.

\section{Data Monitoring Committee}

An independent unblinded data monitoring committee (DMC) will review safety data from cohort 1 to make a recommendation on dose escalation in cohort 2 and review safety data from both cohorts 1 and 2 to make a recommendation on dosing in cohort 3. After completion of phase 2, all data will be unblinded to the DMC and sponsor to determine whether the study should proceed. During phase 3, the DMC will convene to periodically review safety data as well as to review the pre-specified futility interim analysis.

\section{Operational Considerations}

Importantly, ensuring feasibility of study conduct in the setting of the current COVID-19 pandemic was considered during the study design and led to multiple features to accommodate changing institutional practices to limit patient contact to protect medical staff, conserve personal protective equipment, and decrease transmission of infection. This included allowing alternative means of collecting informed consent from potential subjects, limiting the number of blood draws and flexible scheduling, and even permitting longer intravenous (IV) lines to allow the location of IV pumps outside of patient rooms.

\section{DISCUSSION/CONCLUSIONS}

The COVID-19 pandemic has resulted in an unprecedented need for rapid identification and development of effective therapies. Dociparstat, a molecule poised to initiate a phase 3 pivotal study in another indication, was found to be uniquely suited to address the pathophysiology of severe COVID-19. Steps were immediately undertaken to design and initiate a feasible, comparative trial to demonstrate that DSTAT provides added benefit to standard of care in COVID-19 patients at risk of acute lung injury, importantly decreasing their need for ventilatory support and improving overall outcomes.

However, the size and nature of the pandemic presented a variety of operational and scientific challenges. The overwhelming burden on healthcare providers, shortages of personal protective equipment, and the need to limit patient contact by medical personnel to prevent potential transmission of infection have been important considerations in protocol design.

The complexity of treatments being evaluated during the COVID-19 global pandemic has necessitated flexibility in the allowances of concurrent medications and selection of endpoints being evaluated. As the landscape and COVID-19 knowledge base evolve, concurrent medications that would have normally been excluded because of lack of efficacy and added safety concerns (i.e., hydroxychloroquine) had to be considered as potential standards of care in this patient population, with close monitoring of cardiovascular safety. In addition, prophylaxis for DVT was incorporated into the protocol to accommodate institutional practices for anticoagulation in the setting of COVID-19. The US Food and Drug Administration also required inclusion of additional agents approved in other indications with potential benefit in COVID-19 patients (e.g., immunomodulators including IL-6 inhibitors and IL-6 receptor antagonists). This current DSTAT trial in COVID-19 has taken into account those potential confounders and has attempted to minimize their effect on the outcomes being interrogated by close monitoring of cardiovascular safety in the case of 
hydroxychloroquine, protocol-specified dose limitations for DVT prophylaxis and an algorithm for evaluation of potential cases of HIT, and the allowance of non-steroid immunomodulatory agents only in the event of mechanical ventilation, a failure endpoint in the trial. The impact of the recent emergency use authorizations for remdesivir and convalescent plasma and positive clinical outcome data with the addition of dexamethasone on the anticipated effect size of DSTAT has yet to be fully assessed but will be evaluated at the completion of the phase 2 portion of the trial. As these evolving therapies are increasing the proportion of patients surviving without the need for mechanical ventilation, the secondary endpoints of this trial (e.g., the time to improvement in the NIAID ordinal score) will become essential in providing a measure of drug effect on recovery. This identical strategy was utilized by the National Institutes of Health (NIH) in the assessment of remdesivir's activity.

The end goal of this trial is to demonstrate an additive benefit when DSTAT is added to current standard of care therapeutic treatments. The development of new treatments is essential to effectively mitigate the morbidity and mortality in patients by minimizing hospital stays and ensuring the functionality of hospital wards and critical care units.

\section{ACKNOWLEDGEMENTS}

The authors wish to thank in advance all subjects that enroll in this study, enabling collection of important clinical data about the use of DSTAT in the setting of severe COVID-19. In addition, we are grateful to Caryn Barnett (Chimerix, Inc.) and Sarah Oelsner (Scitaris) for their support during the development of this manuscript, and Geraldine Menard, MD (Tulane University Medical Center, New Orleans), for her efforts to enroll the first several subjects into the study.

Funding. Sponsorship for this study and the journal's Rapid Service and Open Access Fees were funded by Chimerix, Inc., located in Durham, NC, USA.

Authorship. All named authors meet the International Committee of Medical Journal Editors (ICMJE) criteria for authorship for this article, take responsibility for the integrity of the work as a whole, and have given their approval for this version to be published.

Authorship contributions. JAL contributed to the design and conduct of the study as well as conceptualization and preparation of the manuscript. JF contributed to the design and conduct of the study, preparation of the manuscript, and was the first investigator to enroll subjects. MEM, RL, ON, TB, and AM contributed to design and conduct of the study and preparation of the manuscript. MEM coordinated all authors' review and revisions of the manuscript and provides medical oversight of the study. All authors read and approved the final manuscript.

Prior Presentation. A non-peer-reviewed version of this article has been available on a preprint server since 04 August 2020 (ResearchSquare: https://www.researchsquare.com/ article/rs-52289/v2).

Disclosures. Joseph A. Lasky is the principal investigator in this Chimerix sponsored study. Jyotsna Fuloria is a participating investigator in this Chimerix sponsored study. Marion E. Morrison, Randall Lanier, Odin Naderer, Tom Brundage, and Allen Melemed are paid employees and shareholders of Chimerix, Inc.

Compliance with ethics guidelines. This trial is designed in accord with the consensus ethical principles derived from international guidelines including the Declaration of Helsinki and Council for International Organizations of Medical Sciences (CIOMS) International Ethical Guidelines; applicable International Council of Harmonization (ICH) Good Clinical Practices (GCP) Guidelines; and applicable laws and regulations. All documents are initially approved by a central institutional review board (Advarra) and/or by the individual site IRB if required. 
Written informed consent is obtained from all participants before any study-related procedures are implemented.

Data availability. The datasets generated during and/or analyzed during the current study are not publicly available as the study is ongoing. When available, the results will be published in a peer-reviewed journal.

Open Access. This article is licensed under a Creative Commons Attribution-NonCommercial 4.0 International License, which permits any non-commercial use, sharing, adaptation, distribution and reproduction in any medium or format, as long as you give appropriate credit to the original author(s) and the source, provide a link to the Creative Commons licence, and indicate if changes were made. The images or other third party material in this article are included in the article's Creative Commons licence, unless indicated otherwise in a credit line to the material. If material is not included in the article's Creative Commons licence and your intended use is not permitted by statutory regulation or exceeds the permitted use, you will need to obtain permission directly from the copyright holder. To view a copy of this licence, visit http://creativecommons.org/licenses/by$\mathrm{nc} / 4.0 /$.

\section{REFERENCES}

1. Wang D, Hu B, Hu C, Zhu F, Liu X, Zhang J, et al. Clinical characteristics of 138 hospitalized patients with 2019 novel coronavirus-infected pneumonia in Wuhan, China. JAMA J Am Med Assoc Am Med Assoc. 2020;323:1061-9.

2. Wu F, Zhao S, Yu B, Chen YM, Wang W, Song ZG, et al. A new coronavirus associated with human respiratory disease in China. Nat Nat Res. 2020;579: 265-9.

3. Xu Z, Shi L, Wang Y, Zhang J, Huang L, Zhang C, et al. Pathological findings of COVID-19 associated with acute respiratory distress syndrome. Lancet Respir Med Lancet Publ Group. 2020;8:420-2.

4. Tian S, Hu W, Niu L, Liu H, Xu H, Xiao SY. Pulmonary pathology of early-phase 2019 novel coronavirus (COVID-19) pneumonia in two patients with lung cancer. J Thorac Oncol. 2020;15: 700-4.

5. Levi M, Thachil J, Iba T, Levy JH. Coagulation abnormalities and thrombosis in patients with COVID-19. Lancet Haematol. 2020;7:e438-40.

6. Tang N, Bai H, Chen X, Gong J, Li D, Sun Z. Anticoagulant treatment is associated with decreased mortality in severe coronavirus disease 2019 patients with coagulopathy. J Thromb Haemost. 2020;18:1094-9.

7. Paranjpe I, Fuster V, Lala A, Russak AJ, Glicksberg BS, Levin MA, et al. Association of treatment dose anticoagulation with in-hospital survival among hospitalized patients with COVID-19. J Am Coll Cardiol. 2020;76:122-4.

8. Lauzier F, Arnold DM, Rabbat C, Heels-Ansdell D, Zarychanski R, Dodek P, et al. Risk factors and impact of major bleeding in critically ill patients receiving heparin thromboprophylaxis. Intensive Care Med. 2013;39:2135-43.

9. Rao NV, Argyle B, Xu X, Reynolds PR, Walenga JM, Prechel M, et al. Low anticoagulant heparin targets multiple sites of inflammation, suppresses heparininduced thrombocytopenia, and inhibits interaction of RAGE with its ligands. Am J Physiol Cell Physiol. 2010;299:C97-110.

10. Chen L, Long X, Xu Q, Tan J, Wang G, Cao Y, et al. Elevated serum levels of S100A8/A9 and HMGB1 at hospital admission are correlated with inferior clinical outcomes in COVID-19 patients. Cell Mol Immunol. 2020;17:992-4.

11. Kim S, Young Kim S, Pribis JP, Lotze M, Mollen KP, Shapiro R, et al. Signaling of high mobility group box 1 (HMGB1) through toll-like receptor 4 in macrophages requires CD14. Mol Med. 2013;19: 88-98.

12. Herold T, Jurinovic V, Arnreich C, Lipworth BJ, Hellmuth JC, Von Bergwelt-Baildon M, Klein M, et al. Elevated levels of IL- 6 and CRP predict the need for mechanical ventilation in COVID-19. J Allergy Clin Immunol. 2020;146(1):128-136.e4.

13. Huang C, Wang Y, Li X, Ren L, Zhao J, Hu Y, et al. Clinical features of patients infected with 2019 novel coronavirus in Wuhan. China Lancet. 2020;395:497-506.

14. Sharma L, Wu J, Patel V, Sitapara R, Rao NV, Kennedy TP, et al. Partially-desulfated heparin improves survival in Pseudomonas pneumonia by enhancing bacterial clearance and ameliorating lung injury. J Immunotoxicol. 2014;11:260-7. 
15. Griffin KL, Fischer BM, Kummarapurugu AB, Zheng S, Kennedy TP, Rao NV, et al. 2-O, 3-O-desulfated heparin inhibits neutrophil elastase-induced HMGB-1 secretion and airway inflammation. Am J Respir Cell Mol Biol. 2014;50:684-9.

16. Zheng S, Kummarapurugu AB, Afosah DK, Sankaranarayanan NV, Boothello RS, Desai UR, et al. 2-O, 3-O desulfated heparin blocks high mobility group box 1 release by inhibition of p300 acetyltransferase activity. Am J Respir Cell Mol Biol. 2017;56:90-8.

17. Fiuza C, Bustin M, Talwar S, Tropea M, Gerstenberger E, Shelhamer JH, et al. Inflammation-promoting activity of HMGB1 on human microvascular endothelial cells. Blood. 2003;101: 2652-60.

18. Nativel B, Marimoutou M, Thon-Hon VG, Gunasekaran MK, Andries J, Stanislas G, et al. Soluble HMGB1 is a novel adipokine stimulating IL-6 secretion through RAGE receptor in SW872 preadipocyte cell line: contribution to chronic inflammation in fat tissue. Chen $\mathrm{X}$, editor. PLoS ONE. 2013;8:e76039.

19. Middleton EA, He X-Y, Denorme F, Campbell RA, Ng D, Salvatore SP, et al. Neutrophil extracellular traps (NETs) contribute to immunothrombosis in COVID-19 acute respiratory distress syndrome. Blood. 2020;136(10):1169-79.

20. Barnes BJ, Adrover JM, Baxter-Stoltzfus A, Borczuk A, Cools-Lartigue J, Crawford JM, et al. Targeting potential drivers of COVID-19: Neutrophil extracellular traps. J Exp Med. 2020;217(6):e20200652.

21. Zuo Y, Yalavarthi S, Shi H, Gockman K, Zuo M, Madison JA, et al. Neutrophil extracellular traps in COVID-19. JCI insight. 2020;5(11):e138999.

22. Kowalska MA, Zhao G, Zhai L, David G, Marcus S, Krishnaswamy $S$, et al. Modulation of protein $C$ activation by histones, platelet factor 4 , and heparinoids: new insights into activated protein $C$ formation. Arterioscler Thromb Vasc Biol. 2014;34: 120-6.

23. Manne BK, Denorme F, Middleton EA, Portier I, Rowley JW, Stubben CJ, et al. Platelet gene expression and function in COVID-19 patients. Blood. 2020;136(11):1317-29.

24. Beigel JH, Tomashek KM, Dodd LE, Mehta AK, Zingman BS, Kalil AC, et al. Remdesivir for the treatment of Covid-19-preliminary report. N Engl J Med. 2020;383:992-4.

25. Harrill AH, Roach J, Fier I, Eaddy JS, Kurtz CL, Antoine DJ, et al. The effects of heparins on the liver: application of mechanistic serum biomarkers in a randomized study in healthy volunteers. Clin Pharmacol Ther. 2012;92:214-20.

26. Joglekar MV, Quintana Diez PM, Marcus S, Qi R, Espinasse B, Wiesner MR, et al. Disruption of PF4/H multimolecular complex formation with a minimally anticoagulant heparin (ODSH). Thromb Haemost. 2012;107:717-25. 